\title{
5T4 Glycoprotein Regulates the Sensory Input-Dependent Development of a Specific Subtype of Newborn Interneurons in the Mouse Olfactory Bulb
}

\author{
Sei-ichi Yoshihara, ${ }^{1}$ Hiroo Takahashi, ${ }^{1}$ Nobushiro Nishimura, ${ }^{1}$ Hiromi Naritsuka, ${ }^{2}$ Taichi Shirao, ${ }^{3}$ Hirokazu Hirai, ${ }^{4}$ \\ Yoshihiro Yoshihara, ${ }^{5}$ Kensaku Mori, ${ }^{2}$ Peter L. Stern, ${ }^{6}$ and Akio Tsuboi ${ }^{1}$ \\ ${ }^{1}$ Laboratory for Molecular Biology of Neural System, Advanced Medical Research Center, Nara Medical University, 840 Shijo-cho, Kashihara, \\ Nara 634-8521, Japan, ${ }^{2}$ Department of Physiology, Graduate School of Medicine, University of Tokyo, Tokyo 113-0033, Japan, ${ }^{3}$ Nikon Instech Company, \\ Ltd., Tokyo 100-0006, Japan, ${ }^{4}$ Department of Neurophysiology, Gunma University Graduate School of Medicine, Gunma 371-8511, Japan, ${ }^{5}$ Laboratory for \\ Neurobiology of Synapse, RIKEN Brain Science Institute, Saitama 351-0198, Japan, and ${ }^{6}$ Cancer Research UK Immunology Group, Paterson Institute for \\ Cancer Research, University of Manchester, Manchester M20 4BX, United Kingdom
}

Sensory input has been shown to regulate development in a variety of species and in various structures, including the retina, cortex, and olfactory bulb $(\mathrm{OB})$. Within the mammalian $\mathrm{OB}$ specifically, the development of dendrites in mitral/tufted cells is well known to be odor-evoked activity dependent. However, little is known about the developmental role of sensory input in the other major $\mathrm{OB}$ population of the GABAgenic interneurons, such as granule cells and periglomerular cells. Here, we identified, with DNA microarray and in situ hybridization screenings, a trophoblast glycoprotein gene, 5T4, whose expression in a specific subtype of OB interneurons is dependent on sensory input. 5T4 is a type I membrane protein, whose extracellular domain contains seven leucine-rich repeats (LRR) flanked by characteristic LRR-N-flanking and C-flanking regions, and a cytoplasmic domain. 5T4 overexpression in the newborn OB interneurons facilitated their dendritic arborization even under the sensory input-deprived condition. By contrast, both 5T4 knockdown with RNAi and $5 T 4$ knockout with mice resulted in a significant reduction in the dendritic arborization of $5 \mathrm{~T}^{+}{ }^{+}$granule cells. Further, we identified the amino acid sequence in the 5T4 cytoplasmic domain that is necessary and sufficient for the sensory input-dependent dendritic shaping of specific neuronal subtypes in the OB. Thus, these results demonstrate that 5T4 glycoprotein contributes in the regulation of activity-dependent dendritic development of interneurons and the formation of functional neural circuitry in the $\mathrm{OB}$.

\section{Introduction}

Neural circuitry is adjusted by sensory input from the external world in postnatal stages. Olfactory bulb $(\mathrm{OB})$ interneurons are a good model for studying neural circuit modification by the sensory experience (Katz and Shatz, 1996; Sanes and Lichtman,

Received Nov. 28, 2011; accepted Dec. 20, 2011.

Author contributions: S.Y. and A.T. designed research; S.Y., H.T., N.N., H.N. and T.S. performed research; T.S., H.H., Y.Y., K.M., and P.L.S. contributed unpublished reagents/analytic tools; S.Y., H.T., N.N., and A.T. analyzed data; S.Y., P.L.S., and A.T. wrote the paper.

This work was supported by grants from Grant-in-Aid for Scientific Research for Basic Research (C) and Priority Areas (Cell Sensor) from the Ministry of Education, Culture, Sports, Science and Technology (MEXT), Japan. A.T. was supported by grants from Naito, Novartis, Terumo, Applied Enzymology, Urakami, Mishimakaiun Memorial, and Uehara Memorial Foundations, and Daiwa Housing Group (Indoor Environmental Medicine), Japan. S.Y. and H.T. were supported by a Grant-in-Aid for Young Scientists (B) from MEXT and a grant from the Kao Foundation for Arts and Sciences, Japan. S.Y. was supported by grants from Sumitomo, Mochida Memorial, Naito, Life Science, Cosmetology, and Takeda Science Promotion Foundations, Japan. H.T. was supported by a Grant-in-Aid for Priority Areas (Neurovascular Network) from MEXT, and grants from Uehara Memorial and Inamori Foundations, Japan. P.L.S. was supported by funding from Cancer Research UK Grant C480/A12328. We gratefully thank H. Sakano for encouraging this study, and the staffs of the animal facility of Nara Medical University for expert assistance. We thank RIKEN BSI Research Resource Center for help in DNA microarray analysis. We gratefully thank the staff of the animal facility and Jian Li of Paterson Institute for Cancer Research for expert contributions.

Correspondence should be addressed to Akio Tsuboi, Laboratory for Molecular Biology of Neural System, Advanced Medical Research Center, Nara Medical University, 840 Shijo-cho, Kashihara, Nara 634-8521, Japan. E-mail: atsuboi@naramed-u.ac.jp.

DOI:10.1523/JNEUROSCI.5907-11.2012

Copyright $\odot 2012$ the authors $\quad 0270-6474 / 12 / 322217-10 \$ 15.00 / 0$
2001; Nithianantharajah and Hannan, 2006). The OB interneurons are generated and integrated into the pre-existing neural circuit throughout life (Lledo et al., 2008; Whitman and Greer, 2009; Adam and Mizrahi, 2010; Kaneko et al., 2010; Sakamoto et al., 2011). Newborn interneurons are generated in the subventricular zone, migrate along the rostral migratory stream (RMS), and differentiate into GABAergic interneurons, such as granule cells (GCs) and periglomerular cells (PGCs) (Lledo et al., 2008; Whitman and Greer, 2009; Adam and Mizrahi, 2010; Kaneko et al., 2010; Sakamoto et al., 2011). It is known that odor-evoked activity affects the survival and integration of newborn OB interneurons (Rochefort et al., 2002; Yamaguchi and Mori, 2005; Lin et al., 2010). In addition, olfactory sensory deprivation or odor-rich environment can promote suppression or acceleration of both dendritic morphogenesis and spine formation of newborn $\mathrm{OB}$ interneurons, respectively (Saghatelyan et al., 2005; Livneh et al., 2009). These results suggest that the odor-evoked activity facilitates the survival rate, dendritic morphogenesis, and spine formation of the OB interneurons. However, little is known about the molecular players or the precise mechanisms that integrate odor-evoked activity with the developmental processes of $\mathrm{OB}$ interneurons.

In this study, DNA microarray and in situ hybridization screenings in the unilaterally naris-occluded OB identified an 
oncofetal glycoprotein gene, 5T4, whose expression in the OB GCs is dependent on sensory input. 5T4 is a type I transmembrane (TM) protein, whose extracellular (EC) domain contains seven leucine-rich repeats (LRR) flanked by characteristic LRR$\mathrm{N}$-flanking and C-flanking regions, and a cytoplasmic domain with a PDZ interacting motif. 5T4 protein was first identified while searching for molecules with invasive properties likely to be shared by placental trophoblasts and cancer cells (Hole and Stern, 1990). 5T4 expression is upregulated in many different carcinomas, while showing only low levels in most normal tissues (Southall et al., 1990) except for high levels in brain and ovary (King et al., 1999; Barrow et al., 2005). The 5T4 overexpression in epithelial cells causes the disruption of cell-cell contacts, downregulation of E-cadherin (Carsberg et al., 1996), altered morphology, and increased cellular motility, while 5T4 upregulation associated with embryonic stem cell differentiation appears to be an integral component of epithelial-to-mesenchymal transition (Eastham et al., 2007; Spencer et al., 2007). Here, using a lentiviral system, providing either gain- or loss-of-function for 5T4 gene in the developmental processes of OB interneurons, plus 5T4 knock-out (KO) mice, we establish that 5T4 glycoprotein regulates the dendritic arborization of the $5 \mathrm{~T}^{+}{ }^{+} \mathrm{GCs}$ in a sensory input-dependent manner.

\section{Materials and Methods}

Mice and naris occlusion procedure. Animal research was approved by the campus committee of Nara Medical University and Paterson Institute for Cancer Research, University of Manchester, and was conducted in accordance with its guidelines. We used ICR male and female mice purchased from Japan SLC. Olfactory sensory deprivation was performed by the naris occlusion. Newborn mice at postnatal day $0(\mathrm{P} 0)$ were anesthetized with ice, and then the unilateral nostril was cauterized by a soldering iron.

$5 T 4$ knock-out mice. We have constructed a $5 T 4 \mathrm{KO}$ mouse line by replacing the second exon of 5T4, which encodes the entire protein, with an IRES-LacZneo reporter gene in ES cells (Southgate et al., 2010). These cells were used to produce chimeric mice and germline progeny; 5T4 heterozygote mice were backcrossed to the C57BL/6 background. The $5 T 4 \mathrm{KO}$ C57BL/6 animals are viable, but adult animals show some structural disorganization within the brain and exhibit a high frequency of hydrocephalus. The frequency of hydrocephalus is $\sim 13 \%$, with the median age of death at $\mathrm{P} 49$.

DNA microarray analysis. Total RNA was extracted separately from the open and closed sides of $\mathrm{OB}$ from three naris-occluded mice (P28). These total RNA samples were used as the probe templates of GeneChip Mouse Genome 430 2.0 DNA microarray (Affymetrix). The DNA microarray expression profiles were compared between the open and closed sides of OB. DNA microarray statistical analysis was performed as described previously (Inoue et al., 2004). The genes expressing a $>1.5$-fold change between the open and closed sides of $\mathrm{OB}$ were further analyzed by in situ hybridization.

Generation and injection of lentiviral vectors. Lentiviral vectors were provided kindly by Dr. Arthur Nienhuis (St. Jude Children's Research Hospital, Memphis, TN) and Dr. Hirokazu Hirai (Gunma University, Maebashi City, Japan). Recombinant lentiviral vectors, expressing the gapEYFP, gapmCherry, and 5T4-IRES-gapEYFP genes under the control of the $C M V$ or $5 T 4$ promoter $(6 \mathrm{~kb})$, were prepared as described previously (Torashima et al., 2006). For 5T4 knockdown experiments, short hairpin RNAs (shRNAs) were expressed under the human $H 1$ promoter. Three sets of shRNAs targeted at the $5 T 4$ gene were designed by siDirect version 2.0 (http://sidirect2.rnai.jp/). The following sets of shRNAs were cloned into the lentiviral vector: sh1, GGTA TCATTACAGATACGATTCAAGAGATCGTATCTGTAATGATACC CTTTTTT; sh2, GACTTTGATCCTTGTTATGTATTCAAGAGATAC ATAACAAGGATCAAAGTCTTTTT; and sh3, GCTCTTACATAGAA CTTTGTATTCAAGAGATACAAAGTTCTATGTAAGAGCTTTTT.

Newborn mice (P0) were anesthetized with ice, and then $0.5 \mu \mathrm{l}$ of lentiviral vector was injected into the lateral ventricle by an Injection
Pump KDS 310 (KD Scientific). The titers of lentivirus were adjusted to $2.0 \times 10^{8}$ transducing units $/ \mathrm{ml}$.

In situ hybridization. The entire coding region of the 5T4 gene was used as the template of a digoxigenin (DIG)-labeled RNA probe. Narisoccluded ICR mice at 3 weeks postnatally (P21) were anesthetized with sodium pentobarbital ( $2.5 \mathrm{mg} / \mathrm{animal})$ and perfused intracardially with $4 \%$ paraformaldehyde (PFA). Mouse brains were dissected out and fixed in PBS containing 4\% PFA on ice for $1 \mathrm{~h}$. Tissues were placed for $1 \mathrm{~d}$ in $30 \%$ sucrose and embedded in O.C.T. compound (Tissue-Tek) in liquid nitrogen. Serial coronal sections $(20 \mu \mathrm{m})$ were cut with a JUNG CM1900 (Leica) and collected on 3-aminopropyl-triethoxysilane-coated slide glasses. The procedures used for hybridization, washings, and antibody reaction were done as described previously (Tsuboi et al., 1999). Slides were incubated with alkaline phosphatase-conjugated anti-DIG antibody (anti-DIG-AP; Roche Diagnostics), and positive cells were stained with NBT/BCIP (Roche Diagnostics) or HNPP Fluorescent Detection Set (Roche Diagnostics). Images were acquired using a BX50 microscope equipped with a CCD camera M3204C (Olympus) and processed by Adobe Photoshop. With the exception of minor adjustments in brightness and contrast, the images were not altered.

Immunohistochemistry. Immunohistochemistry (IHC) of the mouse OB sections was performed as described previously (Yoshihara et al., 2005). Primary antibodies used were as follows: rabbit anti-GFP (1:1000, Life Technologies); rat anti-GFP (1:1000, Nakalai Tesque); rabbit antiRFP (1:1000, Clontech Laboratories); rat anti-RFP (1:1000, ChromoTek); rabbit anti-5T4 antibody (1:1000) (Imamura et al., 2006); sheep anti-5T4 (1:1000, R\&D Systems); mouse anti-mouse5T4 monoclonal antibody (1:1000) (Southgate et al., 2010); and chicken anti-lacZ (1:1000, Abcam). DyLight 488-, DyLight 549-, and DyLight 649-conjugated secondary antibodies were purchased from Jackson ImmunoResearch. In enhanced yellow fluorescent protein (EYFP) IHC, OB sections from ICR mice, where the CMVp-5T4-IRES-gapEYFP lentiviral vector was injected, was enhanced by tyramide signal amplification (TSA) method (PerkinElmer). TSA IHC of OB sections was performed with horseradish peroxidase-conjugated goat anti-rabbit IgG $(\mathrm{H}+\mathrm{L})(1: 1000$, Life Technologies), Alexa Fluor 488-conjugated streptavidin (1:500, Life Technologies), and TSA Biotin Tyramide reagent pack (PerkinElmer). DAPI nuclear counterstaining was performed on all $\mathrm{OB}$ sections. Images were acquired using IX71 microscope equipped with a CCD camera DP30BW (Olympus), a confocal laser microscope FV1000 (Olympus), and a multiphoton confocal laser microscope A1R MP (Nikon). Stacked images in the $x-y$ plane from individual thin-sectioned slices were superimposed with Adobe Photoshop to reveal the entire morphology of cells.

Statistical analyses. Total length of dendrites and 5T4 hybridizingsignal intensities were analyzed with ImageJ software (National Institutes of Health). Total length of dendrites is the sum of all dendritic branches of a single neuron. A branching point is the point where a neurite extends from the cell body or from another neurite. A process has to be at least 10 $\mu \mathrm{m}$ to be considered a branch. The branching number of dendrites is the sum of every branching point from a single neuron. All data were analyzed with Microsoft Excel 2007 using Student's $t$ test. Descriptive statistics were displayed as the mean \pm SEM. Differences were deemed significant at $p<0.05$.

\section{Results}

\section{T4 gene shows sensory input-dependent expression in a} specific subtype of newborn $O B$ interneurons

To test whether sensory input affects dendritogenesis of newborn mouse $\mathrm{OB}$ interneurons, a lentiviral vector, expressing the membrane-associating gapEYFP under the control of the $C M V$ promoter (CMVp-gapEYFP), was injected into the lateral ventricles at $\mathrm{P} 0$ (Fig. $1 \mathrm{~A}$ ), followed by unilateral naris occlusion. After 3 weeks (P21), we analyzed the dendritogenesis of newborn $\mathrm{OB}$ interneurons by confocal microscopy (Fig. $1 B, C$ ) and twophoton microscopy (data not shown). We found the volumes of the external plexiform and GC layers were reduced in the closed side compared with the open side of $\mathrm{OB}$, but variability in the numbers of EYFP ${ }^{+}$interneurons in individual mice precluded 
A
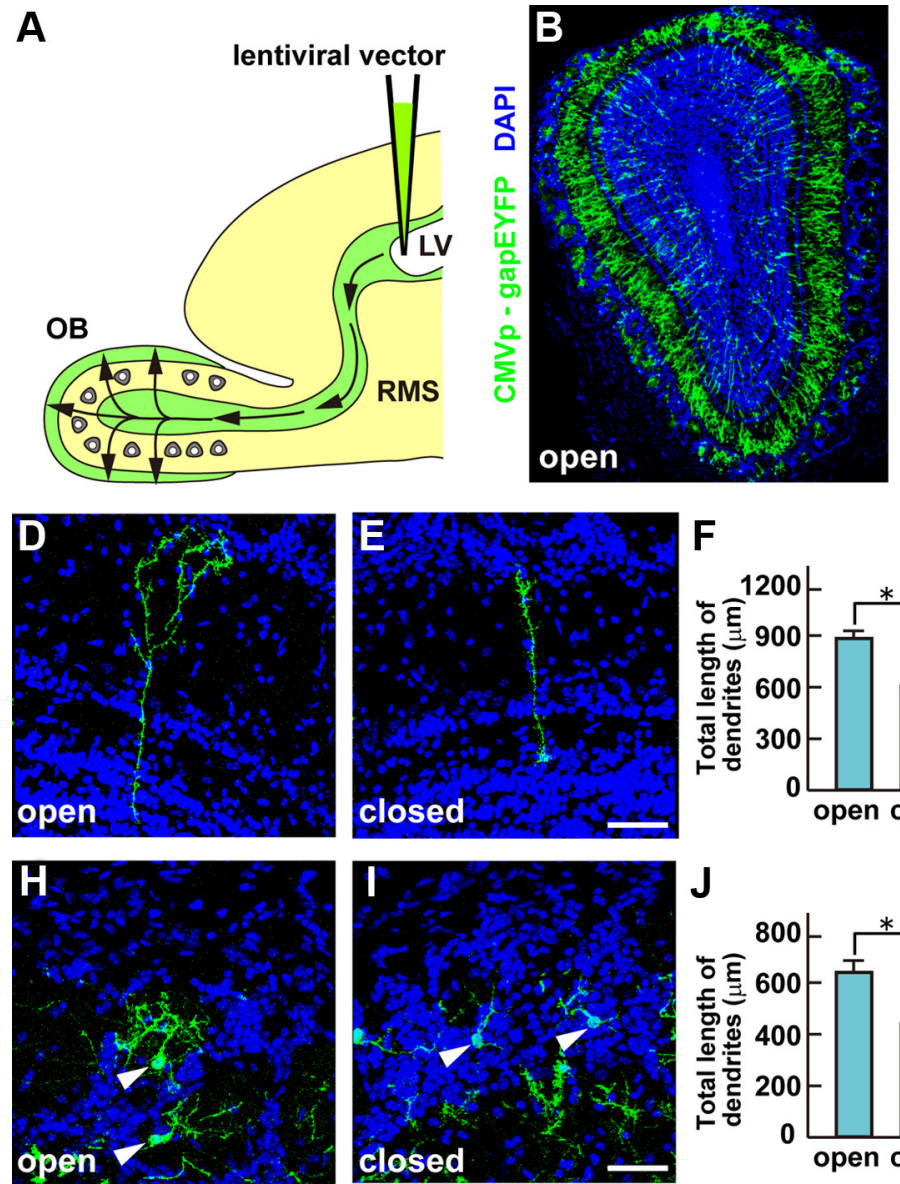

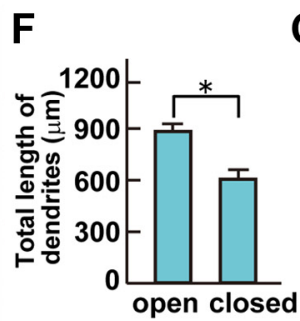

G

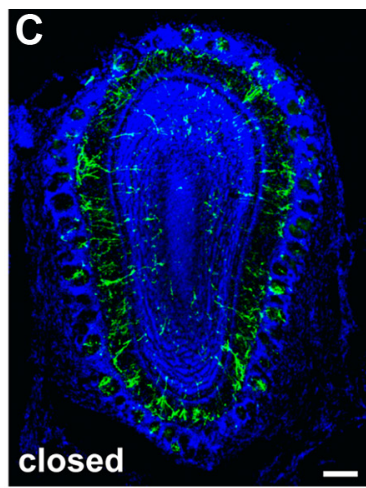

$\mathbf{J}$

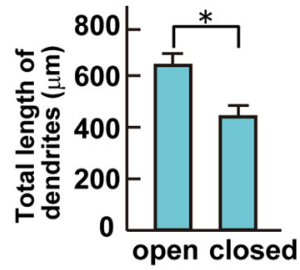

K



Figure 1. Sensory input is required for the dendritic elongation and arborization of newborn $O B$ interneurons. $A$, Schematic diagram of the experimental protocol. A lentiviral vector carrying CMVp-gapEYFP was injected into the lateral ventricle (LV) of PO mice. EYFP ${ }^{+}$interneurons in OB were analyzed 3 weeks (P21) after injection. $\boldsymbol{B}, \boldsymbol{C}$, Newborn $0 B$ interneurons in the open (B) and closed $(C)$ sides of $0 B$ from $P 21$ naris-occluded mice, where the unilateral nostril was closed at $P 0$. The volumes of external plexiform and granule cell layers were reduced on the closed side compared with the open side of OB. Scale bar, $100 \mu \mathrm{m} . \boldsymbol{D}, \boldsymbol{E}$, Newborn $\mathrm{GCS}$ in the open $(\boldsymbol{D})$ and closed $(\boldsymbol{E})$ sides of OB from P21 naris-occluded mice. Scale bar, $50 \mu \mathrm{m}$. $\boldsymbol{F}, \boldsymbol{G}$, Quantification on the total lengths $(\boldsymbol{F})$ and the branching numbers $(\boldsymbol{G})$ of GC dendrites is shown as the mean \pm SEM $\left(\boldsymbol{F}\right.$ : open $=878 \pm 26 \mu \mathrm{m}$, closed $=556 \pm 17 \mu \mathrm{m} ; \boldsymbol{G}$ : open $=3.4 \pm 0.1$, closed $=2.5 \pm 0.1 ;{ }^{*} p<0.01$ compared with the open side, Student's $t$ test, $n=50$ cells in each side of OB from three individuals). $\boldsymbol{H}, \boldsymbol{I}$, Newborn PG(s in the open $(\boldsymbol{H})$ and closed $(\boldsymbol{I})$ sides of $0 \mathrm{~B}$ from P21 naris-occluded mice. Scale bar, $50 \mu \mathrm{m} . J, K$, Quantification of the total lengths $(J)$ and the branching numbers $(K)$ of PGC dendrites is shown as mean values \pm SEM $(J:$ open $=653 \pm 41 \mu \mathrm{m}$, closed $=422 \pm 18 \mu \mathrm{m} ; \boldsymbol{K}:$ open $=$ $17.2 \pm 1.2$, closed $=8.5 \pm 0.8 ;{ }^{*} p<0.01$ compared with the open side, Student's $t$ test, $n=50$ cells in each side of OB from three individuals). Newborn GCs and PGCs had a shorter length and fewer branched dendrites on the closed than the open side of $\mathrm{OB}$.

conclusions about any reduction in numbers of newborn interneurons with naris closure. However, both measurements of the total length and the branching number of GC dendrites (Fig. $1 D-G$ ) from P21 naris-occluded mice showed that there was a 1.6- or 1.4-fold reduction, respectively, in the closed side compared with the open side of OB. We also observed that both the total length and branching number of PGC dendrites were reduced 1.5- and 2.0-fold in the closed side compared with the open side of $\mathrm{OB}$, respectively (Fig. $1 \mathrm{H}-\mathrm{K}$ ). These results confirm that odor-evoked sensory input is necessary for dendritic elongation and arborization of interneurons of GCs and PGCs in $\mathrm{OB}$ (Saghatelyan et al., 2005; Kelsch et al., 2009). To investigate the molecules regulating the development of OB interneurons, a DNA microarray approach was used.

A microarray analysis, searched for genes whose expression levels differed between the open and closed sides of OB but correlated with interneurons (data not shown), was assessed by in situ hybridization. Among 38 candidate genes, $5 \mathrm{~T} 4$ oncofetal trophoblast glycoprotein showed clear differences in expression levels between the open and closed sides of OB (Fig. 2A,B). Interestingly, Imamura et al. (2006) reported that 5T4 is localized in the dendrites of a specific subset of $\mathrm{OB}$ interneurons and is reduced in expression levels by olfactory deprivation. This suggested that $5 \mathrm{~T} 4$ could be a factor regulating the dendritic arborization and synaptic connection of 5T4 ${ }^{+}$GCs, depending on the degree of sensory input. Thus, we chose the 5T4 gene for further analyses to confirm its expression in the $\mathrm{OB}$ and following naris occlusion. 5T4 was expressed in a specific subset of PGCs or GCs at the glomerular layer or at the mitral and superficial GC layers, respectively (Fig. $2 A, B$ ). In the olfactory-deprived OB, $5 \mathrm{~T}^{+}{ }^{+}$cell numbers were decreased 1.9fold in the glomerular, mitral cell, and superficial GC layers (Fig. $2 A-C)$. Moreover, the average fluorescent intensities of $5 T 4$ hybridization signals were reduced 1.7 -fold in the closed side compared with the open side of $\mathrm{OB}$ (Fig. $2 A, B, D$ ). These results demonstrate that $5 T 4$ gene is expressed in the specific subtype of GCs, depending on the sensory input.

Overexpression of $5 \mathrm{~T} 4$ facilitates the dendritic arborization of OB granule cells

Because 5T4 protein is localized preferentially at the GC dendrites in OB (Imamura et al., 2006), it is possible that 5T4 regulates the dendritogenesis or synaptogenesis of $\mathrm{OB}$ interneurons 

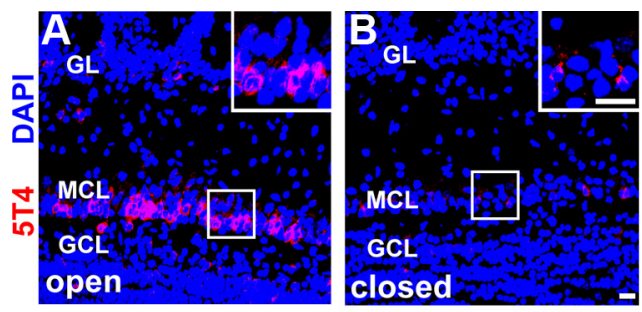

5 T4
N
C


TM PDZ

Figure 2. The $5 T 4$ gene is expressed in a specific subtype of $O B$ interneurons in a sensory input-dependent manner. $A, B$, Fluorescent in situ hybridization of $0 B$ sections from P21 naris-0ccluded mice. $5 T 4$ hybridizing and DAPI staining signals are shown as red and blue, respectively. The $5 T 4$ gene was expressed in a specific subset of PGCs and GCs. The $5 \mathrm{~T} 44^{+}$interneurons were located mainly in the mitral cell layer (MCL) and superficial granule cell layer (GCL), and slightly in the glomerular layer (GL). Insets show the enlarged image of the area enclosed with white squares. Scale bar, 20 $\mu \mathrm{m}$. $\boldsymbol{C}, \boldsymbol{D}$, Quantification on cell numbers $(\boldsymbol{C})$ and hybridizing signal intensities $(\boldsymbol{D})$ of the $5 \mathrm{~T} 4^{+} \mathrm{GCS}$, shown as the mean \pm SEM $\left(\boldsymbol{C}\right.$ : open $=162 \pm 24$ cells $/ 0.01 \mathrm{~mm}{ }^{2}$, closed $=86 \pm 17$ cells $/ 0.01$ $\mathrm{mm}^{2} ; \boldsymbol{D}$ : open $=100 \pm 7 \%$, closed $=58 \pm 5 \% ;{ }^{*} p<0.01$ compared with the open side, Student's $t$ test, $n=50$ cells in each side of 0 B from three individuals). Both the $5 \mathrm{TT}{ }^{+}$cell number and its expression level in each neuron were reduced on the closed side compared with the open side of $0 \mathrm{~B}$.

following sensory input. To investigate the role of $5 \mathrm{~T} 4$ in the neuronal development within $\mathrm{OB}$, a gain-of-function experiment was performed by injecting a lentiviral vector that carries the CMVp5T4-IRES-gapEYFP gene into the lateral ventricles of $\mathrm{P} 0$ mice. After 3 weeks (P21), the dendritogenesis of 5T4-expressing neurons was compared with that of the control EYFP-expressing ones. Differences in the dendritic morphology between the control and the $5 T 4$ overexpressing GCs were dependent on positions of the cell bodies in the layers. For example, in the mitral cell layer, 5T4-overexpressing GCs had 2.6-fold more branched dendrites than those of the control EYFP (Fig. 3A-

$C)$, while in the GC layer they showed 1.5-fold more branched dendrites than the control EYFP (data not shown). 5T4 overexpression in adult GCs following infection into P56 mice gave similar results (a 3.1-fold increase in branching numbers) in those at newborn age (data not shown). These results demonstrate that the 5T4 gain-of-function facilitates the dendritic arborization of newborn GCs in the OB.

When the olfactory sensory input is deprived, both the total length and the branching numbers of GC dendrites are reduced (Fig. 1). We tested whether dendritic arborization could be restored by overexpression of $5 T 4$ under the activity-reduced condition. This was achieved by coinjection of two lentiviral vectors (CMVp-5T4IRES-gapEYFP and control CMVp-gapmCherry) into the lateral ventricles of $\mathrm{P} 0$ mice, followed by unilateral naris occlusion (Fig. $4 A, B$ ). As expected, after 2 weeks (P14), GCs expressing the control $m$ Cherry had 1.6-fold fewer branched dendrites on the closed than on the open side of $\mathrm{OB}$ (Fig. 4C,D, compare controls). Interestingly, GCs overexpressing the 5T4 gene in the mitral cell layer had 2.3- and 3.5 -fold more branched dendrites in both the open and closed sides of $\mathrm{OB}$, respectively (Fig. 4C,D). These results demonstrate that $5 T 4$ overexpression facilitates the dendritic arborization of the $\mathrm{OB}$ interneurons including with olfactory deprivation.

5T4 intracellular domain is necessary and sufficient for the dendritic arborization

A series of lentiviral 5T4 deletion constructs were used to establish which domains of 5T4 are required for the dendritic ar- borization. Interestingly, overexpression of a construct deleting the LRRs of the extracellular domain (5T4- $\Delta$ LRR) gave 2.5 -fold more branched dendrites in the GCs than the control EYFPinjected mice, similar to a 2.6-fold increase seen with the 5T4-full length construct (Fig. $5 B, C, E$ ). By contrast, overexpression of the intracellular (IC) domain-deletion construct (5T4- $\Delta \mathrm{IC}$ ) showed a similar level (1.1-fold) of branching numbers in the GC dendrites to that the control EYFP (Fig. $5 A, D, E$ ). To further map the specific sequence of the 44 aa cytoplasmic domain involved, lentiviral vectors with $10,20,30$, and 40 aa deletions from the $\mathrm{C}$ terminus were generated and injected into $\mathrm{P} 0$ mice (Fig. $5 \mathrm{~B}, \mathrm{~F}-J$ ). Overexpression of the 5 T4 C-terminal 10 aa deletion (5T4- $\Delta \mathrm{C} 10)$ gave 2.6-fold more branched dendrites in the GCs than that of the control EYFP, similar to a 2.6-fold increase seen with overexpression of the $5 T 4$ full length construct (Fig. $5 B, F, J$ ). This 5T4- $\Delta \mathrm{C} 10$ construct lacks the PDZ-binding motif (SDV), showing that it is not involved in the dendritic arborization of GCs. Expression of the 5T4 C-terminal 20 aa deletion (5T4- $\Delta$ C20) gave 1.5 -fold more branching numbers in the GC dendrites than that of the control EYFP (Fig. 5G,J). However, expression of the 5T4 C-terminal 30 or 40 aa deletion construct $(5 \mathrm{~T} 4-\Delta \mathrm{C} 30$ or $5 \mathrm{~T} 4-\Delta \mathrm{C} 40)$ showed a similar level (1.1- and 1.2-fold) of branching numbers in the GC dendrites to that of the control EYFP, respectively (Fig. $5 H-J$ ). These results indicate that the 5T4-IC internal domain without the PDZbinding motif is necessary for the dendritic arborization of GCs.

To test whether the 5T4-IC domain is sufficient for the dendritic arborization, domain swap experiments were performed with CD8, a TM glycoprotein expressed by a subset of T lympho- 

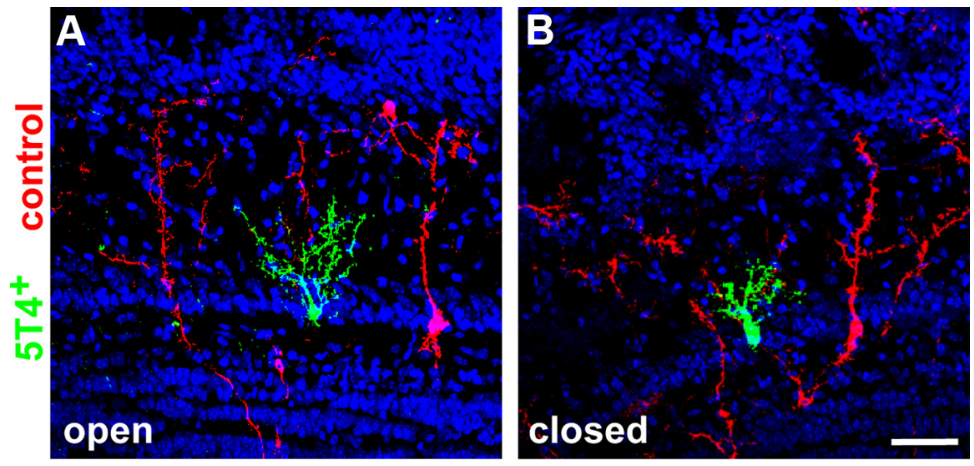

C

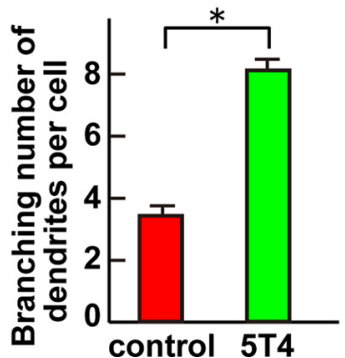

D

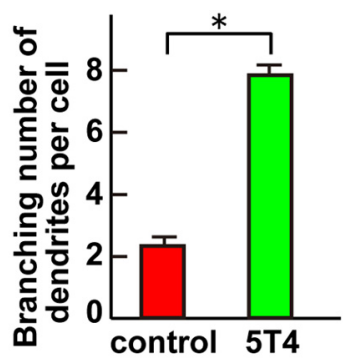

Figure 4. Overexpression of $5 T 4$ restores the dendritic branching of $\mathrm{GCS}$ in the closed side of $0 \mathrm{~B} . \boldsymbol{A}, \boldsymbol{B}$, Coinjection of the 5T4-expressing (CMVp-5T4-IRES-gapEYFP) and control (CMVp-gapmCherry) lentiviral vectors into the lateral ventricles of narisoccluded mice. The 5T4-overexpressing $\mathrm{GCS}\left(5 \mathrm{~T}_{4}{ }^{+}\right)$, where EYFP was also positive, are shown as green; the $m$ Cherry-expressing GCs (control) are shown as red in the open $(\boldsymbol{A})$ and closed $(\boldsymbol{B})$ sides of OB from P14 mice. Scale bar, $50 \mu \mathrm{m}$. C, D, Quantification of branching numbers of the control $\left(\mathrm{mCherry}{ }^{+}\right)$and $5 T 4$-overexpressing $\mathrm{GC}$ dendrites in the open $(\boldsymbol{C})$ and closed $(\boldsymbol{D})$ sides of OB from P14 mice, shown as the mean \pm SEM (C: control $=3.5 \pm 0.2,5 \mathrm{~T} 4=8.0 \pm 0.2 ; \boldsymbol{D}$ : control $=2.2 \pm 0.2,5 \mathrm{~T} 4=7.8 \pm 0.2 ;{ }^{*} p<$ 0.01 compared with the control, Student's $t$ test, $n=30$ cells in each side of $0 B$ from three individuals). The 5T4-overexpressing $\mathrm{GCS}$ showed increased branching numbers of dendrites, even in the closed side of $\mathrm{OB}$.

cyte. A CD8-5T4 chimera comprising the CD8-EC+TM and 5T4-IC domains was generated and injected into P0 lateral ventricles. Ectopic expression of the CMV promoter-driven CD8 full length gave a 1.4-fold increase in branching numbers in the GC dendrites, compared with the control EYFP (Fig. 6A,B,D). By contrast, ectopic expression of the $C M V$ promoter-driven $C D 8-$ $5 T 4$ chimera gave 3.1-fold more branched dendrites in the GCs than that of the control EYFP (Fig. 6A, C,D). These results demonstrate that the 5T4-IC domain is sufficient for the dendritic arborization.

\section{Downregulation of 5T4 leads to reduction of dendritic arborization in endogenous interneurons}

To further investigate the influence of 5T4 in the development of the $5 \mathrm{~T}^{+}{ }^{+} \mathrm{GCs}$, knock-down or dominant-negative lentiviral vectors were used in conjunction with a $5 T 4$ promoter construct to identify interneurons with endogenous 5T4 expression. To evaluate the $5 \mathrm{~T} 4$ promoter containing a $6 \mathrm{~kb}$ region upstream of the initiation codon, the $5 T 4$ promoter $(6 \mathrm{~kb})$-driving gapEYFP (5T4p-gapEYFP) construct was injected into P0 lateral ventricles, and double immunostained with EYFP and 5T4 antibodies at P14. This identified a specific subset of GCs and PGCs, especially in the mitral and superficial GC layers, where EYFP ${ }^{+}$GCs coexpress endogenous $5 T 4$ (Fig. 7A-C, arrowheads). We further analyzed the dendritic morphology only for $\mathrm{EYFP}^{+}$(endogenous $5 \mathrm{~T}^{+}$) in the GCs.

In the $5 T 4$ knockdown studies, we used lentiviral vectors that express three sets of 5T4-shRNAs under the human $H 1$ promoter (H1p-5T4-shRNA). Both the three H1p-5T4-shRNAs and the $5 T 4 p$-gapEYFP vectors were coinjected into $\mathrm{P} 0$ lateral ventricles. Interestingly, coexpression of the 5T4-shRNA and EYFP con- structs gave 2.2-fold fewer branched dendrites in the GCs than that of the control 5T4p-gapEYFP (Fig. $7 D, E, G$ ). This result revealed that the $5 T 4$ knockdown leads to reduction of the dendritic arborization of GCs.

Since the 5T4-IC region is necessary and sufficient for the dendritic arborization of OB interneurons (Figs. 5, 6), overexpression of the 5T4-IC domain alone without the TM domain could sequester the downstream signaling components and block dendritic branching. A lentiviral vector carrying the 5T4p-5T4-IC-IRESgapEYFP gave 1.9-fold fewer branched dendrites than that of the control gapEYFP in the $5 \mathrm{~T}^{+}{ }^{+} \mathrm{GCs}$ (Fig. 7D,F,G). This is consistent with the 5T4-IC region acting as a dominant negative for $5 T 4$ function. Thus, two different types of the $5 T 4$ loss-of-function experiments show the reduction of dendritic arborization in the $5 \mathrm{~T} 4{ }^{+}$granule cells.

We used the CMV promoter in 5T4 overexpression studies in Figures 3-6, since this promoter is robust and can label both $5 \mathrm{~T}^{+}{ }^{+}$and $5 \mathrm{~T} 4{ }^{-}$GCs nearby the mitral cell layer. $5 \mathrm{~T}^{+}{ }^{+} \mathrm{GCs}$ have more branched dendrites $(>6)$ by the endogenous 5 T4 expression than 5T4 ${ }^{-}$GCs (Fig. $7 D, G)$. Thus, the branching numbers of dendrites in the baseline control depend on which GCs [i.e., $\left.5 \mathrm{~T}^{+}{ }^{+}>6\right)$ and $5 \mathrm{~T} 4$ ( $\sim 3)$ ] were labeled by the $C M V$ promoter. However, with the 5 T4 promoter used in Figure 7, we confirmed that 5T4 overexpression facilitates the branching number of dendrites in $5 \mathrm{~T}^{+}$ granule cells (Fig. $8 A, B, D$ ).

The 5T4 EC LRRs were not needed for the dendritic arborization (Fig. $5 B, C, E$ ). So far, the function of the 5T4-EC domain and its ligands/interacting partners has not been reported. However, we found that lentiviral expression of 5T4-EC domain alone resulted in reduction of the branching number of $5 \mathrm{~T}^{+}{ }^{+} \mathrm{GC}$ dendrites (Fig. $8 A, C, D$ ). Thus, it is possible that the dendritic arborization of $\mathrm{OB}$ granule cells may also be influenced by $5 \mathrm{~T} 4$ functions associated with its extracellular domain.

\section{OB granule cells in 5T4 knock-out mice show reduced} dendritic arborization

To further reveal the phenotype of $5 T 4$ loss-of-function in the $\mathrm{OB}$ interneurons, we analyzed the $5 \mathrm{~T}^{+}{ }^{+}$GCs in $5 \mathrm{~T} 4$ knockout mice where the coding region had been replaced by the lacZ gene (Southgate et al., 2010). OB sections of the wild-type $(+/+), 5 T 4$ heterozygous $(+/-)$, and 5T4-null $(-/-)$ mice at P14 were immunostained with 5T4 or LacZ antibody, respectively. In the null mice, the dendrites of $\mathrm{LacZ}^{+}$interneurons seemed to be less extended in the external plexiform layer than those of $5 \mathrm{~T} 4{ }^{+}$interneurons in the wild-type mice (Fig. 9A-F). To confirm this observation, we injected the lentiviral vector carrying $5 T 4$ promoter-driven gapEYFP into lateral ventricles of the $5 \mathrm{~T} 4^{+/+}, 5 \mathrm{~T} 4^{+/-}$, and $5 \mathrm{~T} 4^{-1-}$ mice under the narisoccluded condition. In the open side of OB, $5 \mathrm{~T} 4^{+}\left(\mathrm{EYFP}^{+}\right)$ GCs in the heterozygote showed 1.4-fold less dendritic branching than those in the wild type (Fig. $10 A, B, G$ ), while in 

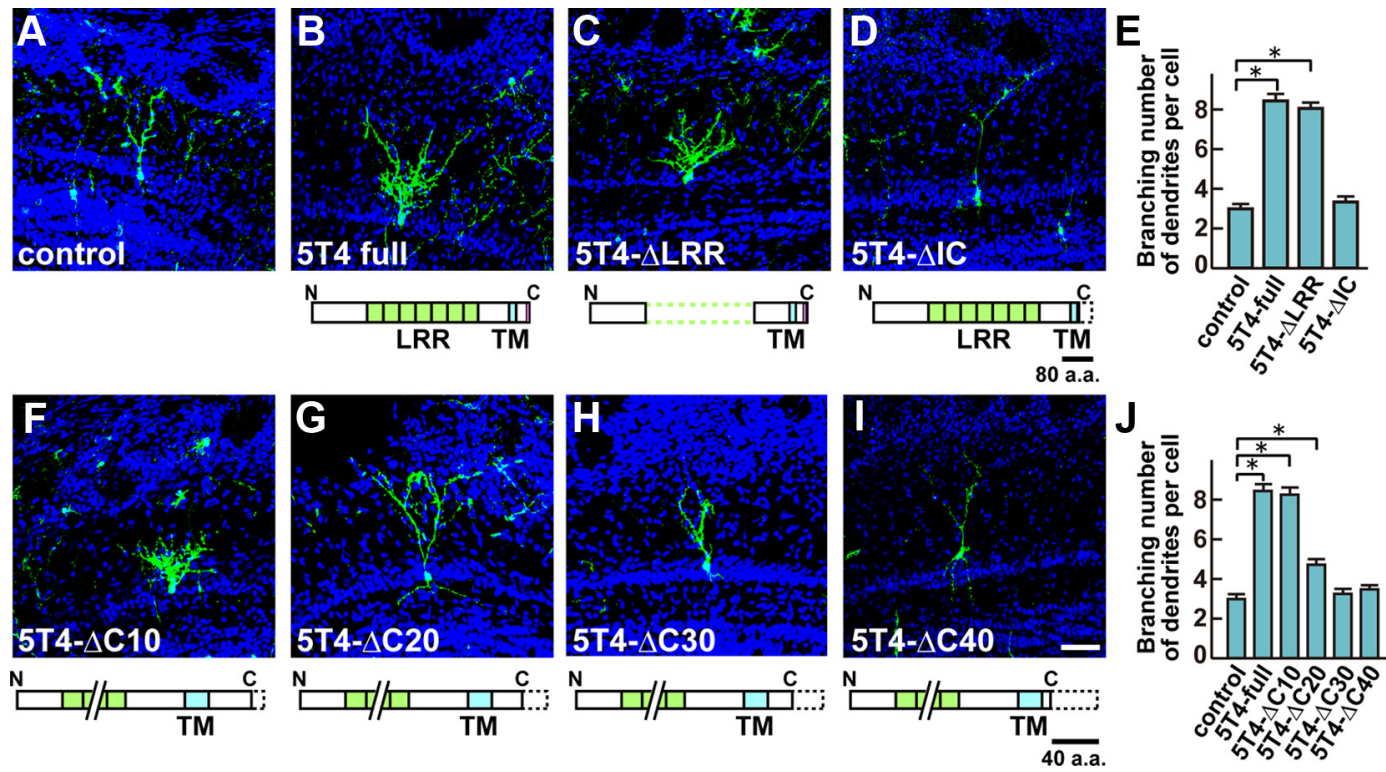

Figure 5. The 5T4 IC region is necessary for the dendritic arborization. $\boldsymbol{A}-\boldsymbol{E}, 5 \mathrm{~T} 4$ deletion experiments. Lentiviral vectors carrying the control $(\boldsymbol{A})$, $5 \mathrm{~T} 4$-full length $(\boldsymbol{B})$, $5 \mathrm{~T} 4-\Delta \mathrm{LRR}(\boldsymbol{C})$, and $5 \mathrm{~T} 4-\Delta \mathrm{IC}$ (D) regions were injected into $\mathrm{PO}$ mice. Note that the $5 \mathrm{~T} 4-\mathrm{IC}$ region is necessary for the dendritic arborization, but the $5 \mathrm{~T} 4$-LRR domain is not. $\boldsymbol{E}, 0$ uantification on branching numbers of $\mathrm{GC}$ dendrites expressing the control, $5 \mathrm{~T} 4$-full length or $5 \mathrm{~T} 4$-deletions in $\mathrm{P} 14 \mathrm{OBs}$, shown as the mean $\pm \mathrm{SEM}$ (control $=3.1 \pm 0.3,5 \mathrm{~T} 4$-full length $=8.2 \pm 0.3,5 \mathrm{~T} 4-\Delta \mathrm{LRR}=7.9 \pm 0.3,5 \mathrm{~T} 4-\Delta \mathrm{IC}=3.4 \pm 0.2$, ${ }^{*} p<0.01$ compared with the control, Student's $t$ test, $n=30$ cells in each type of cells from three individuals). $\boldsymbol{F}$ - $\boldsymbol{J}, 5 \mathrm{~T} 4-\mathrm{IC}$ deletion experiments. Lentiviral vectors carrying a series of 10 aa $(\boldsymbol{F}), 20$ aa $(\boldsymbol{G}), 30$ aa $(\boldsymbol{H})$, and 40 aa $(\boldsymbol{I})$ deletions in the C terminus were injected into P0 mice. Note that the 5T4-IC internal region between 10 and 20 aa in the $C$-terminal end is needed for the dendritic arborization of GCs. Scale bar, $50 \mu \mathrm{m}$. J, Quantification on branching numbers of GC dendrites expressing the sequential 5T4-IC deletions in P14 0Bs, shown as the mean \pm SEM (control $=3.1 \pm$ $0.3,5 \mathrm{~T} 4$-full length $=8.2 \pm 0.3,5 \mathrm{~T} 4-\Delta \mathrm{C} 10=8.1 \pm 0.2,5 \mathrm{~T} 4-\Delta \mathrm{C} 20=4.5 \pm 0.2,5 \mathrm{~T} 4-\Delta \mathrm{C} 30=3.4 \pm 0.2,5 \mathrm{~T} 4-\Delta \mathrm{C} 40=3.6 \pm 0.2{ }^{*} p<0.01$ compared with the control, Student's $t$ test, $n=$ 30 cells in each line from three individuals).

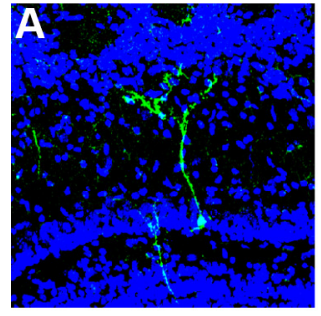

control

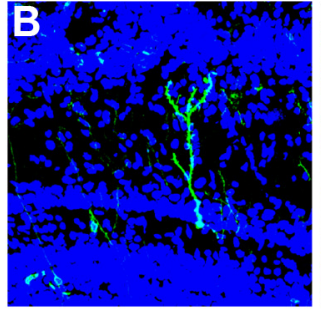

CD8

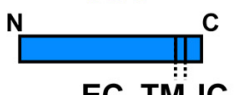

EC TM IC
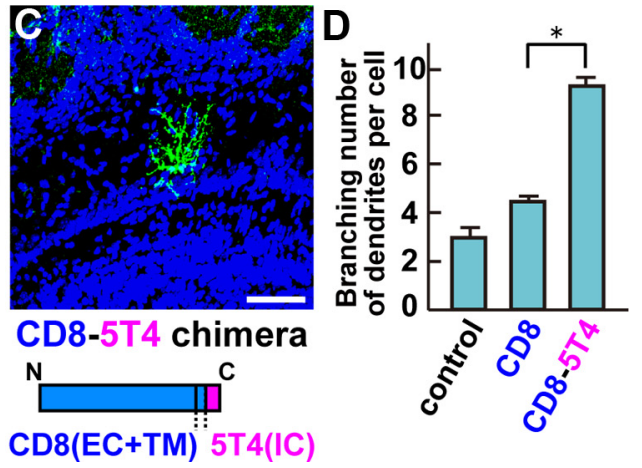

Figure 6. The 5T4IC region is sufficient for the dendritic arborization. $\boldsymbol{A}-\boldsymbol{C}, 5 \mathrm{~T} 4$ domain swap experiments. A lentiviral vector, carrying the control ( $\boldsymbol{A})$, CD8 ( $\boldsymbol{B})$, or $\mathrm{CD} 8-5 \mathrm{~T} 4 \mathrm{Chimera}(\boldsymbol{C})$ comprising the CD8 $(\mathrm{EC}+\mathrm{TM})$ and $5 \mathrm{T4}$ (IC) domains, was injected into P0 lateral ventricles. Expression of the CD8 $-5 \mathrm{~T} 4$ chimera $(\mathbf{C})$ in the $\mathrm{GCS}$ gives rise to more branched dendrites similar to that seen with the $5 \mathrm{T4}$-full length (Fig. $5 B$ ). Scale bar, $50 \mu \mathrm{m}$. D, Quantification on branching numbers of GC dendrites expressing the control, CD8-full length or CD8 $-5 T 4$ chimera in P140Bs, shown as the mean \pm SEM (control $=2.9 \pm 0.2, C D 8=$ $4.1 \pm 0.3, \mathrm{CD} 8-5 \mathrm{~T} 4$ fusion $=8.9 \pm 0.3,{ }^{*} p<0.01$ compared with the control, Student's $t$ test, $n=30$ cells in each line from three individuals).

the null mice the dendrites of $\mathrm{EYFP}^{+}$GCs were 2.3-fold less branched than those in the wild type (Fig. 10A, $C, G$ ). Thus, $5 T 4$ gene dosage appears to correlate with the degree of dendritic arborization. Next, we examined the effect of sensory input on the dendritic branching of $5 \mathrm{~T}^{+}{ }^{+} \mathrm{GCs}$ in the $5 \mathrm{~T} 4$ mutant mice. In the wild-type mice, the branching number of $5 \mathrm{~T} 4^{+}$GCs was reduced 1.9-fold under the naris-occluded condition (Fig. $10 A, D, G$ ), which was consistent with Figure 1. By contrast, the dendritic branching of GCs in the 5T4-null mice showed only slight reduction (1.3-fold) on the closed side than on the open side of OB (Fig. 10C, F, G). These results strongly suggest that $5 \mathrm{~T} 4$ is necessary to regulate the activitydependent dendritic development of $5 \mathrm{~T}^{+}{ }^{+}$granule cells in the $\mathrm{OB}$.

Interestingly, a small number of cell bodies of the $5 \mathrm{~T} 4{ }^{+}$interneurons was located abnormally at the inner GC layer in the 5T4 heterozygous and null mice (Fig. 9G-I). Further, some of these $5 \mathrm{~T} 4{ }^{+}$interneurons had their dendrites in an irregular rather than a radially extended orientation. Since the 5T4 gene is expressed in the mitral and the superficial granule cell layers instead of migrating neuroblasts at the RMS, based on in situ hybridization and immunostaining, this abnormal positioning of $5 \mathrm{~T}^{+}{ }^{+}$interneurons may be derived from a defect of cell migration within OB. These results demonstrate that $5 \mathrm{~T} 4$ is necessary not only for the dendritic arborization, but may also influence the appropriate positioning of the specific subset of $\mathrm{OB}$ interneurons.

\section{Discussion}

A DNA microarray and in situ hybridization approach identified a trophoblast glycoprotein gene, 5T4, as a candidate gene of OB interneuron development with an expression level that depends on the degree of sensory input. The 5T4 gene is expressed in a specific subset of OB interneurons and is required for their mi- 

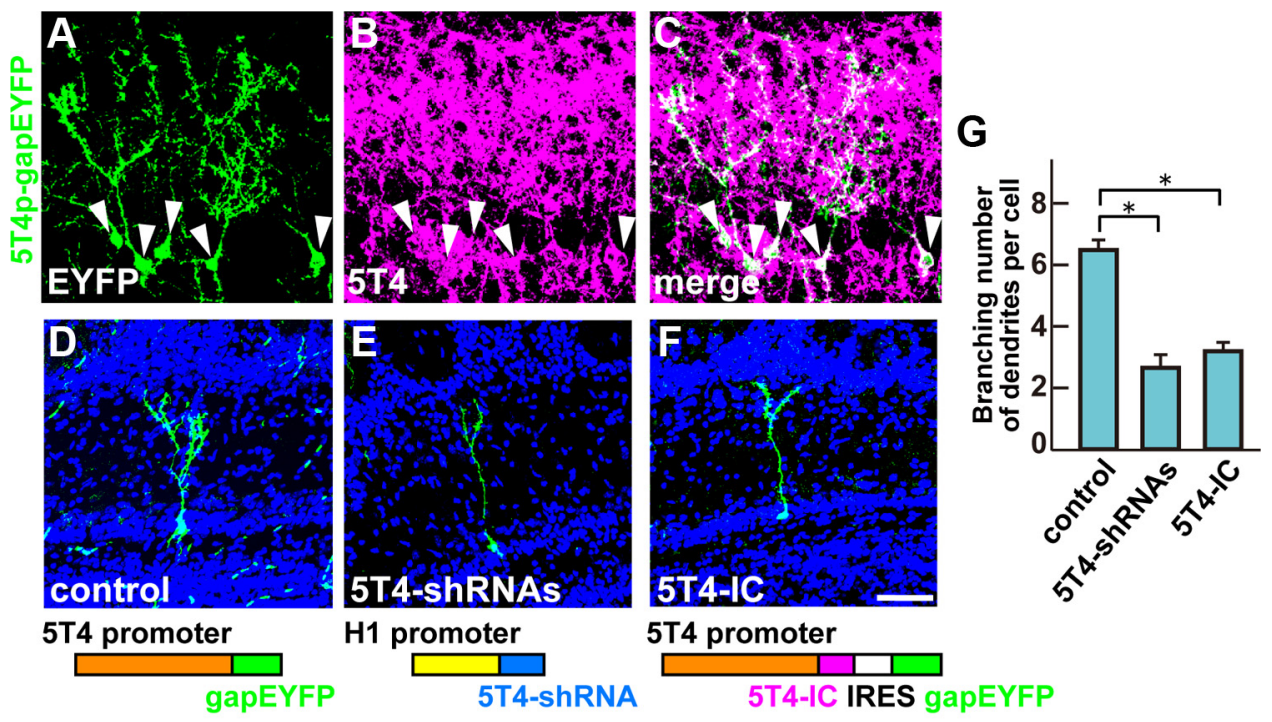

H1 promoter

\section{T4 promoter}

5 T4 promoter

5T4-shRNA

5T4-IC IRES gapEYFP

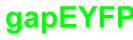

Figure 7. $5 T 4$ knock-down and dominant-negative experiments show reduction of the dendritic arborization in the endogenous cells. $\boldsymbol{A}-\boldsymbol{C}$, The $5 T 4$ promoter analysis. When the $5 T 4$ promoter (6 kb)-driven gapEYFP lentiviral vector was injected into the lateral ventricle, EYFP ${ }^{+} \mathrm{GCS}$ were found at the mitral and granule cell layers in P140B $(A)$. These EYFP ${ }^{+} \mathrm{GCS}$ coexpressed the endogenous $5 T 4$ ( $\boldsymbol{A}-\boldsymbol{C}$, arrowheads). $\boldsymbol{D}-\boldsymbol{F}$, The $5 T 4$ knockdown and dominant-negative experiments. A lentiviral vector carrying the control (D), 5T4-shRNAs $(\boldsymbol{E})$, or 5 TT4-IC $(\boldsymbol{F})$ was injected into P0 lateral ventricles. Expression of the $5 T 4$ shRNAs under the $H 1$ promoter reduced branching numbers of dendrites in the $5 T 4{ }^{+} \mathrm{GCS}(\boldsymbol{E})$ compared with the control (D). Likewise, overexpression of the $5 T 4-\mathrm{IC}$ region gave fewer branched dendrites in the $5 \mathrm{TT}{ }^{+} \mathrm{GCS}(\boldsymbol{F})$. Scale bar, $50 \mu \mathrm{m}$. G, Quantification of branching numbers of $5 \mathrm{~T} 4{ }^{+} \mathrm{GC}$ dendrites expressing the control, $5 \mathrm{~T} 4$-shRNA, or $5 \mathrm{~T} 4-\mathrm{IC}$ in P14 0Bs (control $=6.3 \pm 0.2,5 T 4-\mathrm{RNAi}=2.9 \pm 0.4,5 \mathrm{~T} 4-\mathrm{DN}=3.4 \pm 0.2,{ }^{*} p<0.01$ compared with the control, Student's $t$ test, $n=30$ cells in each line from three individuals).
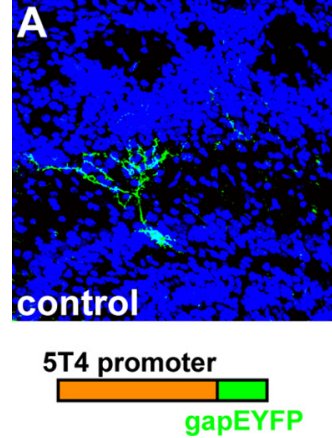
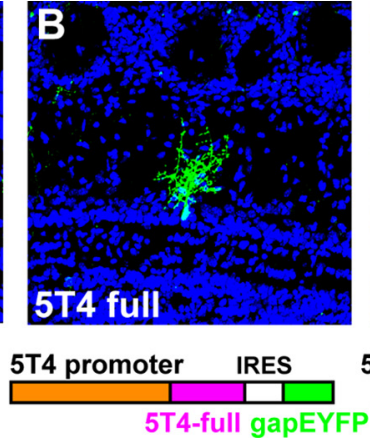

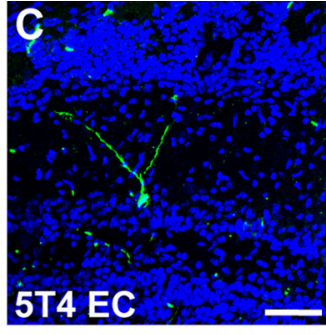

5 T4 promoter IRES

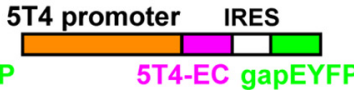

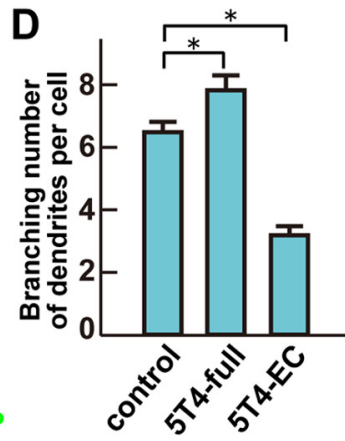

Figure 8. Overexpression of $5 T 4 \mathrm{EC}$ domain shows reduction of the dendritic arborization in the endogenous cells. $A-C$, Overexpression of $5 T 4$-full length or $5 T 4-\mathrm{EC}$ domain in the endogenous $\mathrm{GCS}$. A lentiviral vector carrying the control $(\boldsymbol{A})$, $5 T 4$-full length $(\boldsymbol{B})$, or $5 T 4-\mathrm{EC}(\boldsymbol{C})$ was injected into PO lateral ventricles. Expression of the $5 T 4$-full length under the $5 T 4$ promoter increased branching numbers of dendrites in the $5 \mathrm{~T}^{+}{ }^{+} \mathrm{GCS}(\boldsymbol{B})$ compared with the control $(\boldsymbol{A})$. In contrast, expression of the $5 T 4$ - $\mathrm{EC}$ domain gave fewer branched dendrites in the $5 \mathrm{~T} 4^{+} \mathrm{GCS}(\boldsymbol{C})$ than the control $(\boldsymbol{A})$. Scale bar, $50 \mu \mathrm{m}$. D, Quantification of branching numbers of $5 \mathrm{TT} 4^{+} \mathrm{GC}$ dendrites expressing the control, 574 -full length or $5 \mathrm{~T} 4-\mathrm{EC}$ in P140Bs (control $=6.5 \pm 0.4,5 \mathrm{~T} 4$-full length $=7.8 \pm 0.6,5 \mathrm{~T} 4-\mathrm{EC}=$ $3.2 \pm 0.3,{ }^{*} p<0.01$ compared with the control, Student's $t$ test, $n=30$ cells in each line from three individuals).

gration/positioning, dendritic arborization following sensory input. Comparative studies of $5 \mathrm{~T}^{+/+}, 5 \mathrm{~T} 4^{+/-}$, and $5 \mathrm{~T} 4^{-1-}$ mice showed abnormal phenotypes of both the dendritic branching and the cell positioning of $5 \mathrm{~T} 4{ }^{+}$GCs. These results suggest that 5T4 protein in the neurons is necessary for the precise process of the dendritic arborization and cell positioning/migration.

Sensory input regulates the development of adult-born neurons

Sensory input has been classically recognized as important in shaping development and plasticity throughout the nervous system (Katz and Shatz, 1996; Sanes and Lichtman, 2001; Nithianantharajah and Hannan, 2006). In the mouse olfactory epithelium and bulb, sensory input has been studied during development and in adulthood by a variety of manipulations ranging from odor deprivation to genetic silencing of olfactory sensory neurons (OSNs) (Brunjes, 1994; Zhao and Reed, 2001). Sensory input plays a regulatory role during the OSNs' initial wiring into the glomeruli (Kerr and Belluscio, 2006) as well as in competitive interactions between OSNs as they reinnervate existing glomeruli in adulthood (Zheng et al., 2000; Rochefort et al., 2002; Yamaguchi and Mori, 2005; Imayoshi et al., 2008; Lin et al., 2010; Imamura et al., 2011). In this study, by injecting a lentiviral vector carrying CMVp-gapEYFP gene into the lateral ventricle of unilaterally naris-occluded mice, we found that the sensory input is required for the dendritic elongation and arborization in the OB interneurons (Fig. 1). We also found that the 5T4 gain-of-function and loss-of-function lead to facilitation and reduction of dendritic arborization in the $\mathrm{OB}$ interneurons, respectively, but not dendritic elongation (Figs. 3, 7). These results suggest that the dendritic arborization of $5 \mathrm{~T} 4^{+}$interneurons is augmented in the activated region in $\mathrm{OB}$, where 

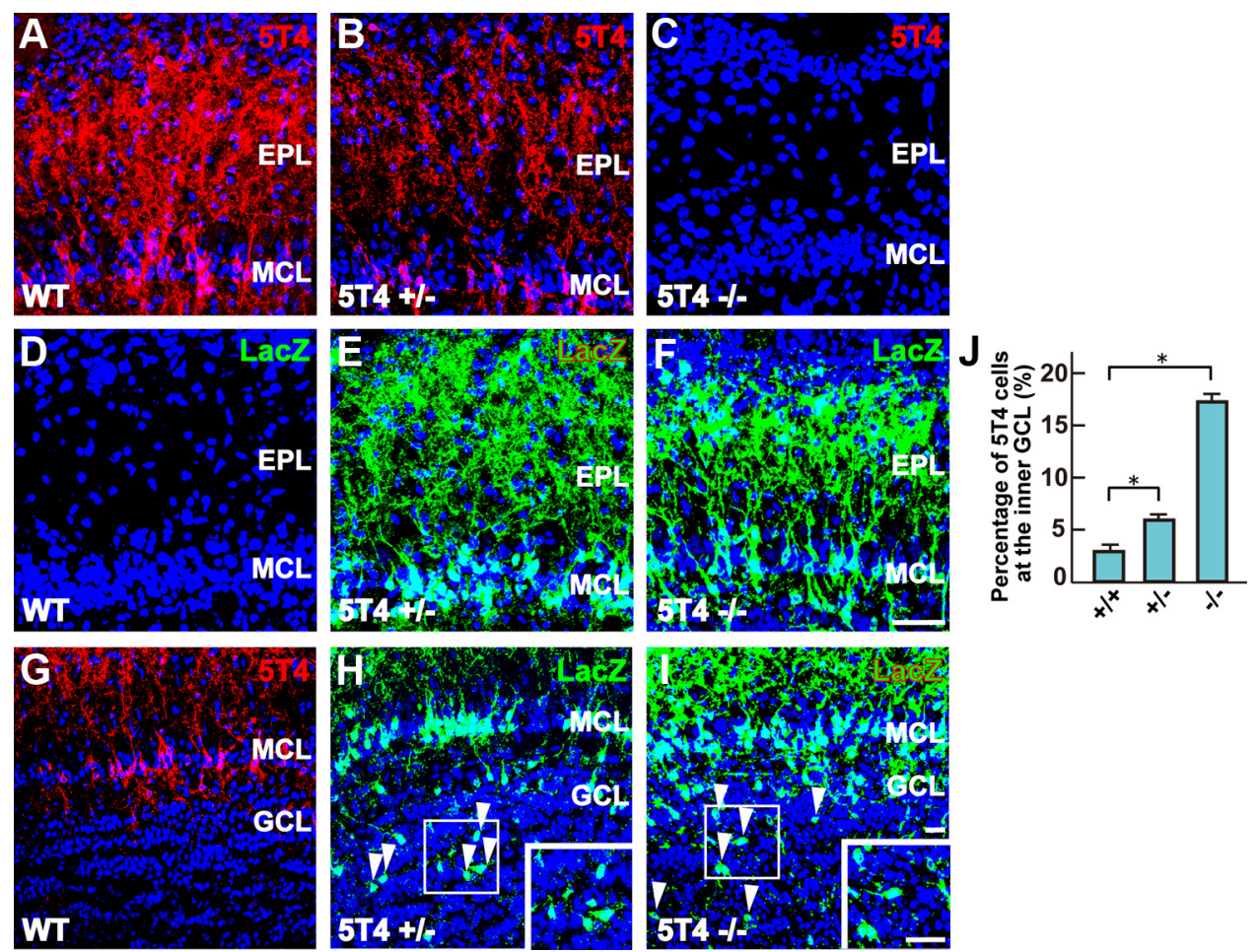

Figure 9. $5 T 4$ knock-out mice show reduction of the dendritic arborization and migration in the corresponding cells. $\boldsymbol{A}-\boldsymbol{F}$, The dendritic arborization of $\mathrm{GCS}$ in the $5 T 4 \mathrm{mutant} 0 \mathrm{Bs}$. $0 \mathrm{~B}$ sections from the WT $(+/+)$, heterozygous $(+/-)$, and null $(-/-)$ mice were immunostained with antibodies against $5 T 4(\boldsymbol{A}-\boldsymbol{C})$ and LacZ $(\boldsymbol{D}-\boldsymbol{F})$, respectively. Scale bar, $50 \mu \mathrm{m}$. $\mathbf{G}-\boldsymbol{I}$, The positioning of $\mathrm{GCS}$ in the $5 T 4$ mutant $0 B$ s. OB sections from the $5 T 4^{+/+}, 5 T 4^{+/-}$, and $5 T 4^{-/-}$mice were immunostained with antibodies against $5 T 4(G)$ and LacZ $(\boldsymbol{H}, I)$, respectively. Insets show the enlarged image of the area enclosed with white squares. Scale bar, $20 \mu \mathrm{m}$. Note that a small number of cell bodies of the $5 \mathrm{TT}{ }^{+}{ }^{+}$interneurons were located abnormally at the inner granule cell layer in the both the $5 T 4^{+/-}$and $5 T 4^{-/-}$mice $\left(\mathbf{G}-I\right.$, arrowheads). $J$, Quantification on percentages of $\mathrm{GC}$ located abnormally at the inner granule cell layer in the $5 T 4^{+/+}, 5 T 4^{+/-}$, and $5 \mathrm{~T}^{-/-}$mice in P14 OBs $\left(5 \mathrm{TH}^{+/+}=2.8 \pm 0.7 \%, 5 \mathrm{~T}^{+/-}=6.1 \pm 0.4 \%, 5 \mathrm{T4^{-/- }}=17.8 \pm 0.6 \%,{ }^{*} p<0.01\right.$ compared with the wild type, Student's $t$ test, data from three individuals in each genotype). EPL, External plexiform layer; MCL, mitral cell layer; GCL, granule cell layer.

the information about abundant odorants in the environment is processed.

How can neural activity in olfactory sensory neurons influence the $5 T 4$ expression in a specific subtype of $\mathrm{OB}$ interneurons?

Specific odorant types activate OSNs expressing the appropriate odorant receptors. These neurons project to specific glomeruli in $\mathrm{OB}$ and subsequently can activate the specific $\mathrm{OB}$ neural circuit locally, upregulating the 5T4 expression level. Our experiments suggest that the increased expression of 5T4 may cause the facilitation of dendritic arborization but not dendritic elongation (Fig. 3). The increased branching numbers of dendrites in $5 \mathrm{~T}^{+}{ }^{+}$interneurons may provide for integrating inputs from a larger receptive field, consistent with the increased 5T4 expression in the OB associated with sensory stimulation. Thus, $5 \mathrm{~T}^{+}{ }^{+}$interneurons that are connected directly or indirectly, via other neurons such as tufted/mitral cells, with glomeruli can be activated by a specific kind of odorants. It is speculated that $5 \mathrm{~T} 4$ plays an important role in both the complexity and refinement of the $\mathrm{OB}$ neural circuitry in an activity-dependent manner.

In cerebral cortex neurons, neural activity is known to induce the elevation of the intracellular calcium ion (Greer and Greenberg, 2008). This calcium elevation activates expression of CAM kinase II and transcription factor CREB. Then, the CREB gene activates several genes, including $B D N F$ and $c$-fos (Greer and Greenberg, 2008). We recently found that the 5T4 promoter of 6 $\mathrm{kb}$ used in this study is downregulated by naris occlusion (data not shown). The $6 \mathrm{~kb}$ region contains putative binding motifs of activity-dependent transcription factors such as $C R E B, E 4 B P 4$, $c$-Ets, GATA-1, C/EBPa, Oct-1, and other genes (data not shown). Further studies on the $6 \mathrm{~kb}$ promoter will lead to elucidating the regulatory mechanism of 5T4 gene expression, depending on the sensory input.

\section{How does 5T4 regulate the dendritic arborization of $\mathrm{OB}$} interneurons?

The 5T4 deletion and domain swap experiments established that the 5T4 intracellular domain is necessary and sufficient for the dendritic arborization of GCs (Figs. 5, 6). It was reported that $5 \mathrm{T4}$ overexpression in the mouse fibroblasts or epithelial cells generates a more spindle-shaped morphology, compared with the control cells with affects on the cytoskeleton organization (Carsberg et al., 1995, 1996). The disruption of the cytoskeleton was associated with the 5T4 cytoplasmic domain, and yeast two-hybrid approaches identified several interacting proteins, through the C-terminal PDZ-binding motif (SDV), such as TIP2/GIPC1 (Awan et al., 2002), that interact with $\alpha$-actinin- 1 to stabilize the actin bundles. This type of functional interaction does not appear relevant to the $\mathrm{OB}$ interneuron development, since GIPC1 is expressed in mitral cells but not GCs and PGCs (data not shown), and the 5T4-PDZ binding motif is not necessary for the dendritic arborization of $\mathrm{OB}$ interneurons (Fig. $5 F, J$ ). Further studies are required to identify the mechanisms underlying the influence of the 5T4 intracellular domain on dendritic arborization. Interestingly, we found that lentiviral expression of 5T4 extra- 

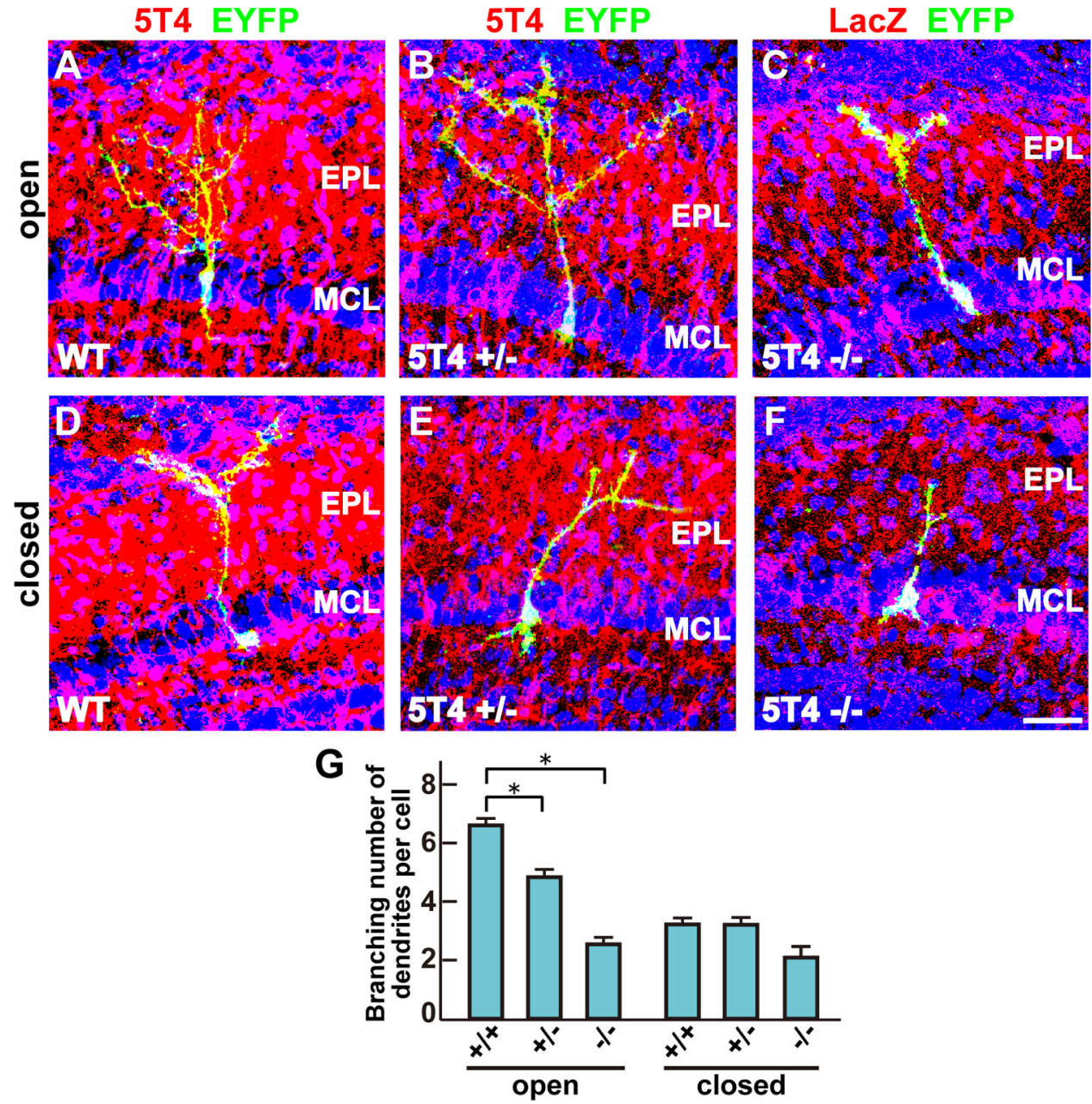

Figure 10. $5 T 4$ knock-out mice show reduction of the dendritic arborization in the corresponding cells. $\boldsymbol{A}-\boldsymbol{F}$, The dendritic arborization of GCs in the $5 T 4$ mutant $0 B s$ under the naris-0ccluded condition. The lentiviral vector carrying $5 T 4$ promoter-driven gapEYFP was injected into lateral ventricles of $5 T 4^{+/+}(\boldsymbol{A}, \boldsymbol{D}), 5 T 4^{+/-}(\boldsymbol{B}, \boldsymbol{E})$, and $5 T 4^{-1-}(\boldsymbol{C}, \boldsymbol{F})$ mice under the naris-0ccluded condition. Scale bar, $30 \mu \mathrm{m}$. G, Quantification on branching numbers of GCs in the $5 T 4$ mutant OBs under the naris-occluded condition (open side: $5 T 4^{+/+}=6.4 \pm 0.5,5 T 4^{+/-}=4.6 \pm 0.6$, $5 T 4^{-1-}=2.8 \pm 0.4$; closed side: $5 T 4^{+/+}=3.3 \pm 0.5,5 T 4^{+/-}=3.1 \pm 0.5,5 T 4^{-1-}=2.1 \pm 0.4{ }^{*} p<0.01$ compared with the wild type, Student's $t$ test, data from 10 cells in each genotype). Note that the dendritic branching of GCS was significantly reduced in the $5 T 4$-null mice compared with the wild type $(A, C, G)$. By contrast, the naris occlusion gave only slight reduction of the branching number of $\mathrm{GC}$ dendrites in the 5T4-null mice compared with the wild type $(\boldsymbol{D}, \boldsymbol{F}, \boldsymbol{G})$.

cellular domain alone resulted in reduction of the branching number of $5 \mathrm{~T} 4{ }^{+}$GCs (Fig. 8A,C). This suggests that 5T4 extracellular domain plays a role in dendritic arborization of OB interneurons via interaction with its ligands.

Cell migration and positioning can also be regulated by the cytoskeleton (Parsons et al., 2010), and in 5T4-null mice there was evidence of imprecise positioning of $5 \mathrm{~T}^{+}{ }^{+}$interneurons in the OB (Fig. 9). However, such effects were not observed in the behavior of interneurons induced by expression of either the shRNA vectors or the dominant-negative construct. It is possible that these neurons are rescued by the endogenous normal $\mathrm{OB}$ microenvironment. Indeed, 5T4 expression is known to facilitate the response to the chemokine CXCL12 through its specific receptor CXCR4, as demonstrated in differentiating mouse embryonic stem cells (Southgate et al., 2010). It is known that CXCR4 regulates cortical interneuron migration and their final laminar positioning (Wang et al., 2011); however, our in situ hybridization and immunostaining failed to detect the CXCR4 transcript and product in the $5 \mathrm{~T} 4{ }^{+} \mathrm{OB}$ interneurons (data not shown).

Recently, it has been shown that 5T4 is both induced by and negatively regulates the Wnt canonical pathway, which then facilitates the response to the noncanonical pathway (Kagermeier-Schenk et al., 2011). Thus, neural activity might induce canonical Wnt ligand production, leading to upregulation of 5T4, which subsequently blocks the canonical and favors the noncanonical pathway in the unconnected OB interneurons. Importantly, disruption of the Wnt5a gene, encoding a noncanonical pathway ligand expressed in a subtype of $\mathrm{OB}$ interneurons, gives rise to reduction of the dendritic extension of GCs, compared with the wild type (Pino et al., 2011). It is possible that Wnt5a production regulates the noncanonical/planar cell polarization pathways, leading to the facilitation of dendritic arborization (Van Amerongen and Nusse, 2009). Since the 5T4-IC internal domain, which lacks the PDZ-binding motif, is necessary for the dendritic shaping in $5 \mathrm{~T}^{+}{ }^{+}$GCs (Fig. $5 F, J$ ), we speculate that it may modulate key interactions that influence Wnt signaling pathways to regulate the dendritic arborization. Identification of proteins that interact with the 5T4 intracellular region will be important on elucidating the mechanisms regulating the dendritic development in OB interneurons, which are likely to include modulation of the cytoskeleton, in the sensory input-dependent manner. 


\section{References}

Adam Y, Mizrahi A (2010) Circuit formation and maintenance-perspectives from the mammalian olfactory bulb. Curr Opin Neurobiol 20:134-140.

Awan A, Lucic MR, Shaw DM, Sheppard F, Westwater C, Lyons SA, Stern PL (2002) 5T4 interacts with TIP-2/GIPC, a PDZ protein, with implications for metastasis. Biochem Biophys Res Commun 290:1030-1036.

Barrow KM, Ward CM, Rutter J, Ali S, Stern PL (2005) Embryonic expression of murine 5T4 oncofoetal antigen is associated with morphogenetic events at implantation and in developing epithelia. Dev Dyn 233:1535-1545.

Brunjes PC (1994) Unilateral naris closure and olfactory system development. Brain Res Brain Res Rev 19:146-160.

Carsberg CJ, Myers KA, Evans GS, Allen TD, Stern PL (1995) Metastasisassociated 5T4 oncofetal antigen is concentrated at microvillus projections of the plasma membrane. J Cell Sci 108:2905-2916.

Carsberg CJ, Myers KA, Stern PL (1996) Metastasis-associated 5T4 antigen disrupts cell-cell contacts and induces cellular motility in epithelial cells. Int J Cancer 68:84-92.

Eastham AM, Spencer H, Soncin F, Ritson S, Merry CL, Stern PL, Ward CM (2007) Epithelial-mesenchymal transition events during human embryonic stem cell differentiation. Cancer Res 67:11254-11262.

Greer PL, Greenberg ME (2008) From synapse to nucleus: calciumdependent gene transcription in the control of synapse development and function. Neuron 59:846-860.

Hole N, Stern PL (1990) Isolation and characterization of 5T4, a tumourassociated antigen. Int J Cancer 45:179-184.

Imamura F, Nagao H, Naritsuka H, Murata Y, Taniguchi H, Mori K (2006) A leucine-rich repeat membrane protein, 5T4, is expressed by a subtype of granule cells with dendritic arbors in specific strata of the mouse olfactory bulb. J Comp Neurol 495:754-768.

Imamura F, Ayoub AE, Rakic P, Greer CA (2011) Timing of neurogenesis is a determinant of olfactory circuitry. Nat Neurosci 14:331-337.

Imayoshi I, Sakamoto M, Ohtsuka T, Takao K, Miyakawa T, Yamaguchi M, Mori K, Ikeda T, Itohara S, Kageyama R (2008) Roles of continuous neurogenesis in the structural and functional integrity of the adult forebrain. Nat Neurosci 11:1153-1161.

Inoue M, Nishimura S, Hori G, Nakahara H, Saito M, Yoshihara Y, Amari S (2004) Improved parameter estimation for variance-stabilizing transformation of gene-expression microarray data. J Bioinform Comput Biol 2:669-679.

Kagermeier-Schenk B, Wehner D, Ozhan-Kizil G, Yamamoto H, Li J, Kirchner K, Hoffmann C, Stern P, Kikuchi A, Schambony A, Weidinger G (2011) The transmembrane protein Waif1/5T4 inhibits Wnt/ $\beta$-catenin signaling and activates noncanonical Wnt pathways by modifying LRP6 subcellular localization. Dev Cell 21:1129-1143.

Kaneko N, Marín O, Koike M, Hirota Y, Uchiyama Y, Wu JY, Lu Q, TessierLavigne M, Alvarez-Buylla A, Okano H, Rubenstein JL, Sawamoto K (2010) New neurons clear the path of astrocytic processes for their rapid migration in the adult brain. Neuron 67:213-223.

Katz LC, Shatz CJ (1996) Synaptic activity and the construction of cortical circuits. Science 274:1133-1138.

Kelsch W, Lin CW, Mosley CP, Lois C (2009) A critical period for activitydependent synaptic development during olfactory bulb adult neurogenesis. J Neurosci 29:11852-11858.

Kerr MA, Belluscio L (2006) Olfactory experience accelerates glomerular refinement in the mammalian olfactory bulb. Nat Neurosci 9:484-486.

King KW, Sheppard FC, Westwater C, Stern PL, Myers KA (1999) Organisation of the mouse and human 5T4 oncofoetal leucine-rich glycoprotein genes and expression in foetal and adult murine tissues. Biochim Biophys Acta 1445:257-270.

Lin CW, Sim S, Ainsworth A, Okada M, Kelsch W, Lois C (2010) Genetically increased cell-intrinsic excitability enhances neuronal integration into adult brain circuits. Neuron 65:32-39.
Livneh Y, Feinstein N, Klein M, Mizrahi A (2009) Sensory input enhances synaptogenesis of adult-born neurons. J Neurosci 29:86-97.

Lledo PM, Merkle FT, Alvarez-Buylla A (2008) Origin and function of olfactory bulb interneuron diversity. Trends Neurosci 31:392-400.

NithianantharajahJ,HannanAJ (2006) Enrichedenvironments, experiencedependent plasticity and disorders of the nervous system. Nat Rev Neurosci 7:697-709.

Parsons JT, Horwitz AR, Schwartz MA (2010) Cell adhesion: integrating cytoskeletal dynamics and cellular tension. Nat Rev Mol Cell Biol 11:633-643.

Pino D, Choe Y, Pleasure SJ (2011) Wnt5a controls neurite development in olfactory bulb interneurons. ASN Neuro 3:e00059.

Rochefort C, Gheusi G, Vincent JD, Lledo PM (2002) Enriched odor exposure increases the number of newborn neurons in the adult olfactory bulb and improves odor memory. J Neurosci 22:2679-2689.

Saghatelyan A, Roux P, Migliore M, Rochefort C, Desmaisons D, Charneau P, Shepherd GM, Lledo PM (2005) Activity-dependent adjustments of the inhibitory network in the olfactory bulb following early postnatal deprivation. Neuron 46:103-116.

Sakamoto M, Imayoshi I, Ohtsuka T, Yamaguchi M, Mori K, Kageyama R (2011) Continuous neurogenesis in the adult forebrain is required for innate olfactory responses. Proc Natl Acad Sci U S A 108:8479-8484.

Sanes JR, Lichtman JW (2001) Induction, assembly, maturation and maintenance of a postsynaptic apparatus. Nat Rev Neurosci 2:791-805.

Southall PJ, Boxer GM, Bagshawe KD, Hole N, Bromley M, Stern PL (1990) Immunohistological distribution of 5T4 antigen in normal and malignant tissues. Br J Cancer 61:89-95.

Southgate TD, McGinn OJ, Castro FV, Rutkowski AJ, Al-Muftah M, Marinov G, Smethurst GJ, Shaw D, Ward CM, Miller CJ, Stern PL (2010) CXCR4 mediated chemotaxis is regulated by $5 \mathrm{~T} 4$ oncofetal glycoprotein in mouse embryonic cells. PLoS One 5:e9982.

Spencer HL, Eastham AM, Merry CL, Southgate TD, Perez-Campo F, Soncin F, Ritson S, Kemler R, Stern PL, Ward CM (2007) E-cadherin inhibits cell surface localization of the pro-migratory 5T4 oncofetal antigen in mouse embryonic stem cells. Mol Biol Cell 18:2838-2851.

Torashima T, Yamada N, Itoh M, Yamamoto A, Hirai H (2006) Exposure of lentiviral vectors to subneutral $\mathrm{pH}$ shifts the tropism from Purkinje cell to Bergmann glia. Eur J Neurosci 24:371-380.

Tsuboi A, Yoshihara S, Yamazaki N, Kasai H, Asai-Tsuboi H, Komatsu M, Serizawa S, Ishii T, Matsuda Y, Nagawa F, Sakano H (1999) Olfactory neurons expressing closely linked and homologous odorant receptor genes tend to project their axons to neighboring glomeruli on the olfactory bulb. J Neurosci 19:8409-8418.

van Amerongen R, Nusse R (2009) Towards an integrated view of Wnt signaling in development. Development 136:3205-3214.

Wang Y, Li G, Stanco A, Long JE, Crawford D, Potter GB, Pleasure SJ, Behrens T, Rubenstein JL (2011) CXCR4 and CXCR7 have distinct functions in regulating interneuron migration. Neuron 69:61-76.

Whitman MC, Greer CA (2009) Adult neurogenesis and the olfactory system. Prog Neurobiol 89:162-175.

Yamaguchi M, Mori K (2005) Critical period for sensory experiencedependent survival of newly generated granule cells in the adult mouse olfactory bulb. Proc Natl Acad Sci U S A 102:9697-9702.

Yoshihara S, Omichi K, Yanazawa M, Kitamura K, Yoshihara Y (2005) Arx homeobox gene is essential for development of mouse olfactory system. Development 132:751-762.

Zhao H, Reed RR (2001) X inactivation of the OCNC1 channel gene reveals a role for activity-dependent competition in the olfactory system. Cell 104:651-660.

Zheng C, Feinstein P, Bozza T, Rodriguez I, Mombaerts P (2000) Peripheral olfactory projections are differentially affected in mice deficient in a cyclic nucleotide-gated channel subunit. Neuron 26:81-91. 